\title{
TIC dirigidas a la superación de barreras educativas de las personas con discapacidad
}

\section{Resumen}

La siguiente revisión bibliográfica tiene como objetivo establecer cuáles Tecnologías de la Información y la Comunicación (TIC) se pueden implementar en los centros educativos para potenciar la superación de barreras de aprendizaje y participación en las personas con discapacidad (PcD), según literatura especializada en el tema. Para ello, se efectúa una revisión bibliográfica en diversas bases de datos que brinden validez y vialidad al tema en estudio; implementando criterios de selección para determinar aquellos documentos que responden mejor al propósito mencionado.

A partir de esta pesquisa, se establecen resultados en los que se aprecia cómo el uso de las TIC puede eliminar o disminuir la presencia de barreras que entorpecen el proceso de desarrollo de este colectivo; resaltando la trascendencia de la implementación de la tecnología en los procesos de mediación pedagógica para el logro de la accesibilidad, inclusión y equidad en el entorno formativo.

Finalmente, se logran establecer conclusiones relacionadas a la forma en que las TIC son fundamentales para la superación de barreras educativas, resaltando la importancia de que éstas brinden respuesta a las características individuales de cada persona; a su vez, se logra determinar cuál herramienta debe utilizarse de acuerdo con las condiciones de discapacidad intelectual, auditiva, visual y física.

Palabras claves: Tecnologías de la información y la comunicación; personas con discapacidad; apoyos educativos; barreras; accesibilidad.

\section{Abstract \\ ICT aimed at overcoming educational barriers for people with disabilities}

The following bibliographical review aims to establish which Information and Communication Technologies (ICT) can be implemented in schools to promote the overcoming of barriers to learning and participation in people with disabilities (PcD), according to specialized literature on the topic. To do this, a bibliographic review is carried out in various databases that provide validity and thoroughness to the subject under study; implementing selection criteria to determine those documents that best respond to the aforementioned purpose.

Based on this research, results are established in which it can be seen how the use of ICT can eliminate or reduce the presence of barriers that hinder the development process of this group; highlighting the transcendence of the implementation of technology in the processes of pedagogical mediation for the achievement of accessibility, inclusion and equity in the training environment.

Finally, conclusions are reached related to the way in which ICTs are fundamental for overcoming educational barriers, highlighting the importance of providing answers to the individual characteristics of each person; in turn, it is possible to determine which tool should be used according with the conditions of intellectual, auditory, visual and physical disability.

Key words: Technology of the information and communication; people with disabilities; educational supports; barriers; accessibility. 


\section{INTRODUCCIÓN}

A nivel global se ha generado una evolución en cuanto a la educación de las personas con discapacidad $(\mathrm{PcD})$, progresando de ideales de exclusión y segregación hacia una sociedad que respeta la diversidad, mediante el ejercicio de la inclusividad, igualdad y equidad; sin embargo, a pesar del avance que se ha logrado alcanzar todavía existen barreras en el aprendizaje y la participación de este colectivo (Campaña Mundial por la Educación, 2013).

Es importante mencionar que actualmente rige el modelo social de la discapacidad, el cual considera que ésta "es una construcción social, [...] que limita e impide que las personas con discapacidad se incluyan, decidan o diseñen con autonomía su propio plan de vida en igualdad de oportunidades" (Victoria, 2013, p.1093); por lo que desde esta perspectiva, es indispensable brindar herramientas que respondan a las características de cada individuo, para garantizar equidad en cuanto al acceso al aprendizaje y a las posibilidades de estimular el desenvolvimiento autónomo de las PcD.

En el ámbito educativo, el tema de las TIC ha sido ampliamente tratado alrededor del mundo; pues éstas han venido a representar herramientas significativas para la superación de barreras, la accesibilidad, inclusión y el desarrollo de entornos formativos dinámicos e interactivos, que promueven la creatividad y el pensamiento crítico en el estudiantado.

Ante esto y bajo la perspectiva de que los usos de las TIC "son múltiples y se distribuyen prácticamente en todos los campos de la actividad humana" (Sánchez, 2013, p. 17), es fundamental investigar su implementación como apoyos y herramientas motivadoras para que el estudiantado con discapacidad participe de forma plena en su proceso educativo; brindando una educación universal y equitativa, que ofrezca mayores oportunidades de aprendizaje a cada persona.

Por esta razón, en la presente revisión bibliográfica se responderá al problema de investigación ¿cuáles TIC se pueden implementar en las escuelas y colegios para potenciar la superación de barreras de aprendizaje y participación en las PcD?, todo esto según literatura especializada en el tema; para ello se efectuó una búsqueda de fuentes bibliográficas, que permitan identificar los diferentes usos que tienen las TIC en los procesos formativos y su función en el desarrollo pleno de esta población.

Ante esto, el objetivo principal de esta revisión bibliográfica es brindar ejemplos que puedan utilizar las PcD, para garantizar la superación de barreras que enfrente este colectivo; por lo que, a partir de dicha pesquisa, se pretende dar a conocer la importancia que tiene la implementación de las TIC en los procesos de mediación pedagógica, para estimular las habilidades y destrezas que les permita ser partícipes activos dentro de la sociedad.

\section{LITERATURA SOBRE EL TEMA}

En el transcurrir de los años se ha generado una evolución en cuanto a la concepción de la discapacidad, la cual en la actualidad se rige bajo el modelo social; esta perspectiva, se basa en ideales de inclusión, equidad, respeto a la diversidad y superación de barreras para la participación plena.

Desde este modelo, la discapacidad es el resultado de las barreras que le presenta la sociedad a la persona para desenvolverse con autonomía; por lo que, hoy en día se pretende brindar los apoyos y estimular competencias que le permitan a los individuos actuar con empoderamiento y autodeterminación en los diversos entornos (Victoria, 2013). 
El Poder Ejecutivo de Costa Rica (2018), identifica diversas condiciones de discapacidad caracterizadas por las barreras físicas, psicosociales o sensoriales que se le presentan al individuo al interactuar con el entorno, como lo son la discapacidad intelectual, auditiva, visual y física o motriz.

Ante las diversas condiciones de discapacidad existentes, en el ámbito educativo es elemental brindar los apoyos y herramientas fundamentales para posibilitar el desarrollo integral de la población con discapacidad; siendo esencial, comprender los apoyos "como recursos y estrategias con el objetivo de promover el desarrollo, la educación, los intereses y el bienestar de una persona y mejorar el funcionamiento individual" (AMRR, 2002; citado por Fundación Juan Ciudad, 2016, p. 18), convirtiéndose en instrumentos para la superación de barreras y el alcance de entornos accesibles.

A consecuencia, las TIC han venido a manifestarse como apoyos importantes en el establecimiento de oportunidades de equidad e inclusividad; pues como indican Molina \& Cuevas (2014), éstas

han revolucionado la sociedad moderna, hoy son instrumentos imprescindibles para el desarrollo humano y para alcanzar una mejor calidad de vida. Esta realidad es constatable para el común de la gente y particularmente para las personas con necesidades especiales. La ciudadanía en general se ha visto afectada positivamente con el uso de estas herramientas, pero el efecto en las personas con discapacidad ha sido significativamente superior. (p. 4).

De acuerdo con lo anterior, las TIC son herramientas innovadoras que posibilitan la superación de barreras, el estímulo de la autodeterminación y el empoderamiento, lo que fortalece el actuar autónomo de las PcD; convirtiéndose en apoyos esenciales a implementar en el trabajo realizado con esta población.

Cabe resaltar que, "las nuevas tecnologías son las herramientas más potentes al alcance del profesorado en cualquier tipo de enseñanza, por lo tanto, el aprendizaje del alumnado se ve motivado, participa, se implica y muestra su interés (García, 2014, p. 4); razón por la cual, en la actualidad las TIC han venido a representar elementos e instrumentos enriquecedores para los procesos formativos dirigidos a los niños, niñas, jóvenes y adultos con discapacidad.

Por otra parte, en la elaboración del presente documento se identificaron artículos de revistas, libros y tesis que sirvieron como base para desarrollar el tema en estudio, encontrando antecedentes fundamentales acerca del funcionamiento de las TIC como herramientas de apoyo para la superación de barreras en entornos educativos. En la siguiente tabla se muestran los hallazgos recopilados.

TABLA 1

Literatura cuyo tema es las herramientas de apoyo para la superación de barreras educativas

\begin{tabular}{|c|c|c|c|c|}
\hline \multicolumn{5}{|c|}{ Referencias encontradas sobre el tema, ordenadas cronológicamente } \\
\hline Autor & Documento & Año & Lugar & Tipo \\
\hline $\begin{array}{l}\text { Daniel Zappalá, } \\
\text { Andrea Köppel y } \\
\text { Miriam Suchodolski }\end{array}$ & $\begin{array}{l}\text { Inclusión de TIC en escuelas para alumnos } \\
\text { con discapacidad intelectual }\end{array}$ & 2011 & Argentina & Libro \\
\hline $\begin{array}{l}\text { Mayka García García y } \\
\text { Rafael López Azuaga }\end{array}$ & $\begin{array}{l}\text { Explorando, desde una perspectiva } \\
\text { inclusiva, el uso de las TIC para atender } \\
\text { a la diversidad }\end{array}$ & 2012 & España & Revista \\
\hline Pere Marqués Graells & $\begin{array}{l}\text { Impacto de las TIC en la educación: } \\
\text { funciones y limitaciones }\end{array}$ & 2012 & España & Libro \\
\hline $\begin{array}{l}\text { Jaime Ribeiro y } \\
\text { Sergio Sánchez Fuentes }\end{array}$ & Inclusión Educativa a través de las TIC & 2013 & España & Revista \\
\hline
\end{tabular}




\begin{tabular}{|c|c|c|c|c|}
\hline \multicolumn{5}{|c|}{ Referencias encontradas sobre el tema, ordenadas cronológicamente } \\
\hline Autor & Documento & Año & Lugar & Tipo \\
\hline $\begin{array}{l}\text { Claudia Serrano Ruíz, } \\
\text { Carolina Ramírez Ramírez, } \\
\text { Jheidy Abril Miranda, } \\
\text { Lizeth Ramón Camargo, } \\
\text { Linda Guerra Urquijo y } \\
\text { Nathali Clavijo González }\end{array}$ & $\begin{array}{l}\text { Barreras contextuales para la participación } \\
\text { de las personas con discapacidad física }\end{array}$ & 2013 & Colombia & Revista \\
\hline $\begin{array}{l}\text { Keilin Molina Fallas y } \\
\text { Felisa Cuevas Cordero }\end{array}$ & $\begin{array}{l}\text { TIC y Educación de Personas con } \\
\text { Discapacidad en Costa Rica }\end{array}$ & 2014 & Costa Rica & Libro \\
\hline María Luisa de Miguel García & $\begin{array}{l}\text { Las TICs aplicadas a las Necesidades } \\
\text { Educativas Especiales }\end{array}$ & 2014 & España & $\begin{array}{l}\text { Trabajo final de } \\
\text { graduación }\end{array}$ \\
\hline $\begin{array}{l}\text { Marisol Rodríguez Correa y } \\
\text { María José Arroyo González }\end{array}$ & Las TIC al servicio de la inclusión educativa & 2014 & España & Revista \\
\hline Paula Muñoz Álvarez & $\begin{array}{l}\text { Inventario de recursos TIC para niños con } \\
\text { necesidades educativas especiales }\end{array}$ & 2014 & Guatemala & Tesis \\
\hline $\begin{array}{l}\text { Jorge Herrando Garijo y } \\
\text { Rosa María Coco Martín }\end{array}$ & $\begin{array}{l}\text { Papel de las TIC en personas } \\
\text { con baja visión }\end{array}$ & 2015 & España & Tesis \\
\hline Claudia Rodríguez Barrera & $\begin{array}{l}\text { Uso de las TIC para favorecer el proceso } \\
\text { de aprendizaje de estudiantes con } \\
\text { Discapacidad Intelectual en la Institución } \\
\text { Educativa Nicolás Gómez Dávila, Bogotá, } \\
\text { Colombia. Estudio de caso }\end{array}$ & 2015 & México & Tesis \\
\hline $\begin{array}{l}\text { Tatiana Valencia Molina, } \\
\text { Andrea Serna Collazos, } \\
\text { Solanlly Ochoa Angrino, } \\
\text { Adriana Caicedo Tamayo, } \\
\text { Jairo Montes González, } \\
\text { José Chávez Vescance }\end{array}$ & $\begin{array}{l}\text { Competencias y estándares TIC desde la } \\
\text { dimensión pedagógica: Una perspectiva } \\
\text { desde los niveles de apropiación de las TIC } \\
\text { en la práctica educativa docente }\end{array}$ & 2016 & Colombia & Libro \\
\hline $\begin{array}{l}\text { José Fernández Batanero, } \\
\text { Miguel Reyes Rebollo y } \\
\text { Mohammed El Homran }\end{array}$ & $\begin{array}{l}\text { TIC y discapacidad. Principales barreras } \\
\text { para la formación del profesorado }\end{array}$ & 2018 & España & Revista \\
\hline $\begin{array}{l}\text { Sonia Romero Martínez, } \\
\text { Irene Gonzáles Calzada, Ana } \\
\text { García Sandoval y } \\
\text { Alicia Lozano Domínguez }\end{array}$ & $\begin{array}{l}\text { Herramientas tecnológicas para la } \\
\text { educación inclusiva }\end{array}$ & 2018 & España & Revista \\
\hline
\end{tabular}

Fuente: Elaboración propia basado en Zappalá, Köppel y Suchodolski (2011), García \& López (2012), Marqués (2012), Ribeiro \& Sánchez (2013), Sánchez (2013), Serrano, Ramírez, Abril, Ramón, Guerra, \& Clavijo (2013), Molina \& Cuevas (2014), García (2014), Rodríguez \& Arroyo (2014), Muñoz (2014), Herrando \& Coco (2015), Rodríguez (2015), Vásquez (2015), Valencia, Serna, Ochoa, Caicedo, Montes \& Chávez (2016), Fernández, Reyes \& El Homran (2018) y Romero, Gonzáles, García \& Lozano (2018).

Todos estos documentos, contienen investigaciones sobre el uso de las TIC en la educación y su contribución en la superación de barreras por parte de las PcD. Es elemental comprender que "la integración de las TIC en los procesos de enseñanza y aprendizaje actualmente es aceptada y realizada por muchas instituciones y docentes" (Valencia, Serna, Ochoa, Caicedo, Montes y Chávez, 2016, p. 8); utilizándose en los centros educativos como medio de innovación y apoyo para el desarrollo de competencias en el estudiantado. 


\section{MÉTODO}

\section{Procedimiento desarrollado para la localización de las fuentes consultadas}

Diseño: con el objetivo de recabar información veraz, se efectuó una revisión bibliográfica acerca del tema de las TIC como herramientas de apoyo para la superación de barreras de las PcD.

Estrategia de búsqueda: se efectúa una pesquisa de información en bases de datos como la del Instituto de Monterrey, Dialnet, Scielo y Google Académico, así como la revista Científica de FAREM-Estelí y libros de la editorial de la Universidad Estatal a Distancia, logrando efectuar un mayor acercamiento al tema de investigación mediante la recopilación de tesis, artículos de revista y libros. También se implementaron diversos criterios para que la selección de fuentes sea eficaz y se pudiera obtener datos certeros que le proporcionen validez y fiabilidad a la presente revisión bibliográfica.

Criterios de inclusión y exclusión: se utilizaron una serie de pautas para determinar la inclusión de documentos, como lo son la indagación y comprobación la procedencia de la información, verificar si el texto lo ha escrito un autor que sea reconocido en esa área, observar la fecha de publicación y que ésta no sobrepase los ocho años de antigüedad; todo esto para recolectar datos actualizados y confiables.

Análisis de los datos: a partir de los datos recopilados se brinda sustento teórico sobre cómo las TIC son fundamentales para la superación de barreras educativas, incluyendo su papel en el proceso de aprendizaje y brindando ejemplos de TIC a utilizar según la condición de discapacidad de cada individuo.

\section{RESULTADOS}

\section{Las TIC para la superación de barreras en la educación}

Las PcD a lo largo del tiempo han tenido que enfrentar una serie de situaciones que han impedido su participación dentro de la sociedad, éstas barreras han sido definidas por Vásquez (2015) como "todas aquellas limitaciones, obstáculos y dificultades que el entorno establece para que la persona, independientemente de su condición, pueda desenvolverse de manera plena, integral y aprovechando al máximo sus capacidades" (p. 8); por lo que pueden entorpecer el desarrollo integral de este colectivo.

Igualmente, autores como Serrano, Ramírez, Abril, Ramón, Guerra \& Clavijo (2013), consideran que las barreras son "factores que obstaculizan la participación y el desarrollo de las personas, ya que tienen un impacto directo, tanto en [...] las estructuras corporales, como en la realización de las actividades de la vida diaria y en el desempeño social" (p. 42); siendo fundamental brindar apoyos y oportunidades que permitan la superación de las mismas.

Bajo esta línea, es fundamental considerar el aporte que han hecho las TIC a la sociedad y como éstas abren un sinfín de posibilidades que se transcriben en herramientas de apoyo, para que las PcD obtengan mayores oportunidades de aprendizaje y estimulen habilidades y competencias que deriven en su desenvolvimiento autónomo; favoreciendo así el alcance de una mejor calidad de vida.

Aunado a lo anterior, Fernandez, Reyes \& El Homran (2018) mencionan que las TIC proveen beneficios a las PcD, esto al "facilitar el acceso a todo tipo de información de manera más fácil y cómoda; favorecer la autonomía de los estudiantes, pudiéndose adaptar a las necesidades y demandas de cada alumno 
o alumna" (p. 8), representando un elemento enriquecedor en los procesos de formativos dirigidos a este colectivo.

A su vez, De la Torre \& Domínguez (2012) reconocen la importancia de la implementación de las TIC dentro de la oferta educativa, estableciendo que éstas

brindan una serie de posibilidades verdaderamente importantes al proceso enseñanza aprendizaje. Dentro de ella se pueden mencionar la eliminación de las barreras espacio-temporales entre el profesor y el estudiante, la flexibilización de la enseñanza, la ampliación de la oferta para el estudiante, que favorecen el aprendizaje cooperativo y el autoaprendizaje. (p. 94).

Lo anterior, permite evidenciar cómo las TIC representa herramientas para la superación de barreras y elementos esenciales para el estímulo de entornos formativos que motiven el aprendizaje, aumenten las oportunidades de participación y estimulen la autonomía en el estudiantado. A continuación, se visualiza un esquema que abarca el pensamiento de varios autores con respecto a la importancia de las TIC para la superación de barreras educativas.

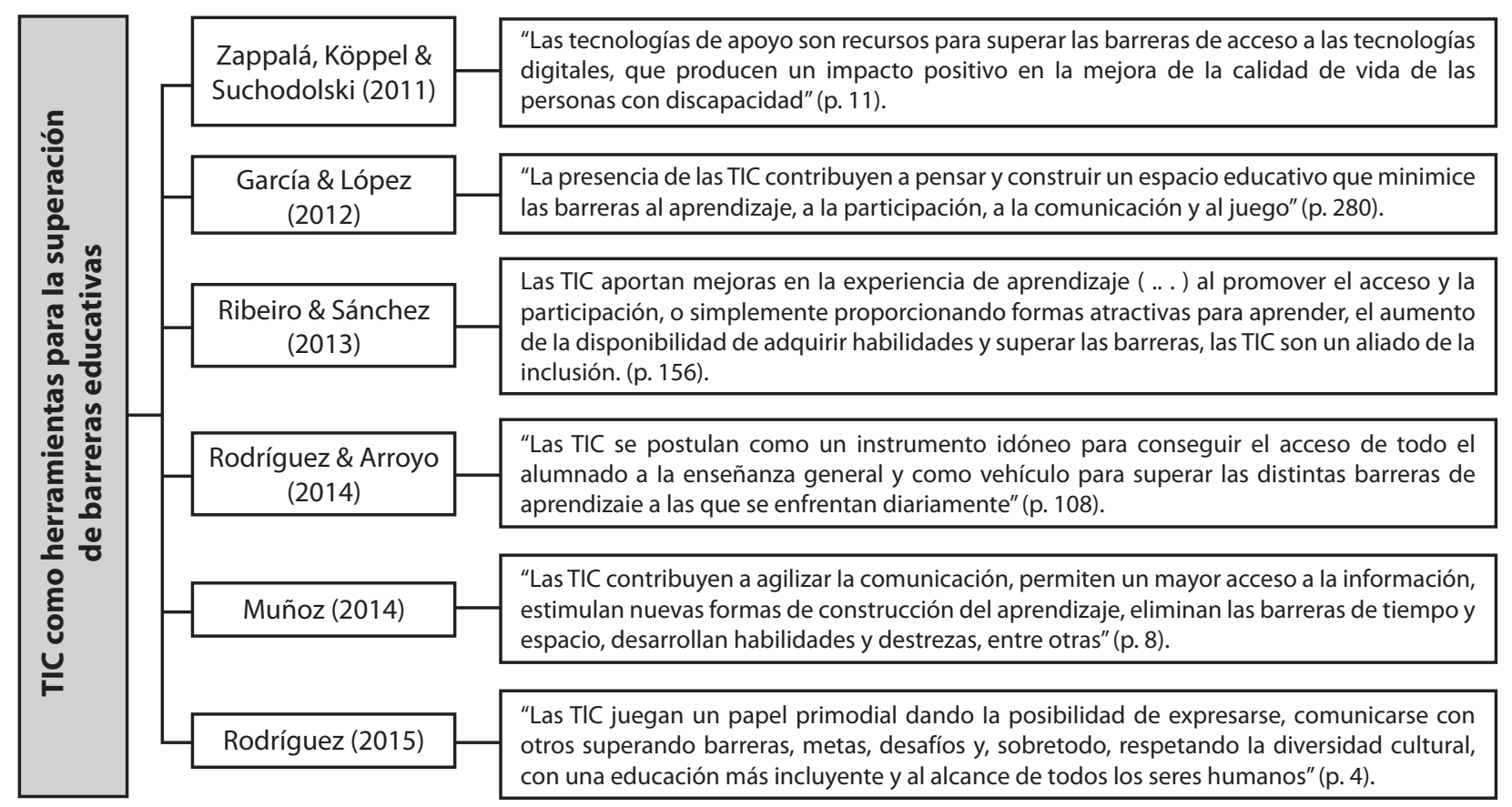

Figura 1. TIC para la superación de barreras educativas. Fuente: Elaboración propia basada en Zappalá, Köppel \& Suchodolski (2011), García \& López (2012), Ribeiro \& Sánchez (2013), Rodríguez \& Arroyo (2014), Muñoz (2014) y Rodríguez (2015).

En la figura anterior, se evidencia como al implementar las TIC en los centros educativos se contribuye en la eliminación de barreras de aprendizaje; garantizando que los estudiantes tengan acceso a herramientas tecnológicas que fortalezcan su participación plena e impulsen el desarrollo de habilidades y destrezas dirigidas a la vida independiente.

Al respecto, García (2014) menciona que las TIC permiten efectuar varias actividades a la vez y satisfacer la curiosidad humana, lo que conlleva al alcance de una vida plena; ante esto, la implementación de las mismas por parte de las PCD fomentará la eliminación las barreras, el desarrollo de empoderamiento y el actuar independiente de cada individuo. 


\section{Papel de las TIC en el proceso de aprendizaje de las PCD}

Las TIC sin duda han transformado el contexto de la educación, al ofrecer aportes a los docentes que propician el establecimiento de oportunidades de aprendizaje de calidad al estudiantado, promoviendo diversas metodologías en cuanto al diseño, aplicación y evaluación los procesos de enseñanza, tomando en cuenta las características de cada persona.

Valencia et al. (2016), indican que "los usos de las TIC en la educación pueden favorecer los procesos de enseñanza y aprendizaje orientados a la construcción de aprendizajes significativos" (p. 9), facilitando la adquisición de nuevos conocimientos y aportando así beneficios en el aprendizaje de los estudiantes.

Así mismo, Marqués (2012) considera que existen múltiples razones para utilizar las TIC dentro del ámbito educativo, las cuales se identificarán en la siguiente figura:

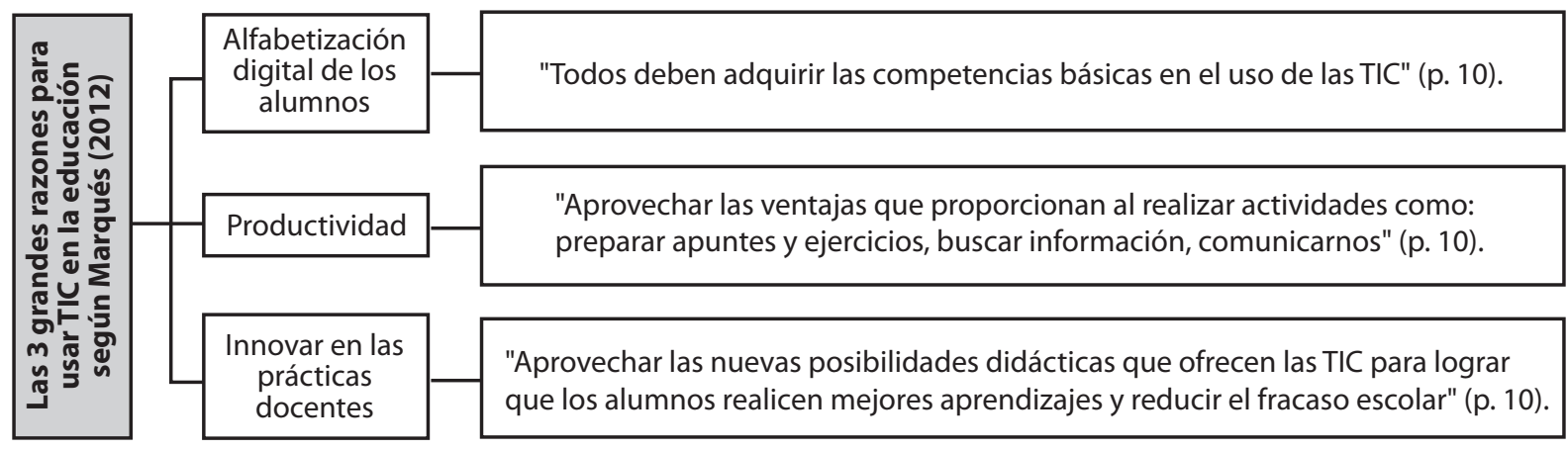

Figura 2. Razones para utilizar las TIC dentro del ámbito educativo. Fuente: Elaboración propia basada en Marqués (2012).

Según lo anterior, la implementación de las TIC en los centros educativos fomenta la motivación e interactividad, pues sus usos "en la educación pueden favorecer los procesos de enseñanza y aprendizaje orientados a la construcción de aprendizajes significativos" (Valencia et al., 2016, p. 9); siendo elemental implementarlas como herramientas innovadoras que capten la atención del estudiantado.

Por lo tanto, al aprovechar el potencial tecnológico se puede transformar el ambiente educativo en un escenario rico en procesos pedagógicos, ya que "las nuevas tecnologías son las herramientas más potentes al alcance del profesorado en cualquier tipo de enseñanza, por lo tanto, el aprendizaje del alumnado se ve motivado, participa, se implica y muestra su interés" (García, 2014, p. 1); por lo cual se debe utilizar distintos tipos de TIC que colaboren en la construcción de mejores aprendizajes.

A raíz de lo anterior, se comprende que las TIC cumplen una función enriquecedora dentro de la mediación pedagógica; al permitir que la población estudiantil desarrolle de forma eficaz habilidades y destrezas que favorezcan las competencias básicas, dirigidas al alcance de la autonomía de las PcD.

Por otra parte, los centros educativos deben ofrecer un ambiente en el que los individuos puedan interactuar con seguridad e independencia, utilizando herramientas tecnológicas que potencien las competencias que van a requerir para efectuar un rol activo dentro de la sociedad (Sánchez, 2013).

Es importante mencionar que, las TIC pueden llegar a cumplir una función de rampa, las cuales les permitirá al estudiantado empoderarse del aprendizaje a través de apoyos debidamente seleccionados, respondiendo a sus habilidades y características individuales; lo que contribuirá a la disminución de barreras educativas que se puedan presentar. 
la armonía en la utilización de las rampas digitales se produce, no sólo por su calidad técnica y prestaciones, sino, sobre todo, porque proporcionan un proceso de comunicación entre el alumno, la familia y la comunidad, lo cual facilita un enriquecimiento mutuo en este mundo diverso y tecnológico que esperamos sea, también, más inclusivo. (p. 81).

Lo anterior, infiere a que las TIC benefician la inclusión de las PCD a los contextos sociales, educativos, laborales y familiares; posibilitando el establecimiento de puentes de comunicación que favorezcan el desarrollo pleno y por tanto mejoren la calidad de vida de este colectivo.

Cabe señalar que, el implementar TIC dentro del aula conlleva múltiples beneficios, potencializando la formación de competencias dirigidas al desenvolvimiento exitoso en los diversos ámbitos; al respecto, García (2014) establece una serie de habilidades que las PCD pueden desarrollar al implementar estas herramientas como parte de su proceso educativo.

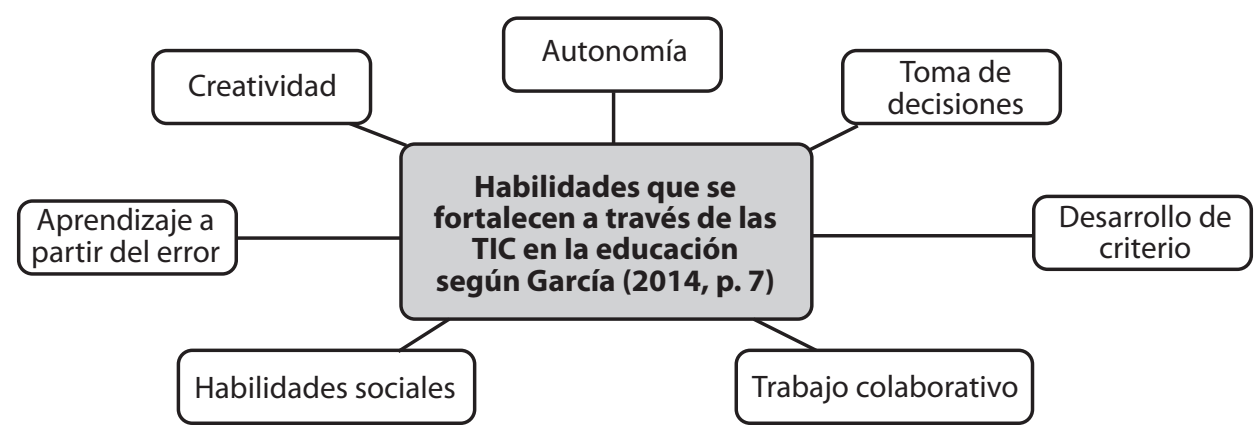

Figura 3. Habilidades que las PCD pueden desarrollar al implementar TIC. Fuente: Elaboración propia basada en García (2014).

Según lo anterior, las TIC posibi an el fortalecimiento de habilidades necesarias para el desenvolvimiento autónomo; por lo que, identificar las herramientas tecnológicas adecuadas a las características individuales de cada persona, estimulará competencias dirigidas a la futura inclusión sociolaboral de esta población.

Cabe resaltar que, las TIC forman "parte del diario vivir y son un apoyo que permiten potencializar los aprendizajes en los diferentes ámbitos generando cambios en la forma de relacionarse entre sí y el contexto en el que se desenvuelven día a día" (Rodríguez, 2015, p. 9); esto al impulsar la autonomía e independencia en la vida cotidiana de las PcD.

\section{Ejemplos de TIC a utilizar según la condición de discapacidad}

Las TIC representan herramientas importantes en el ámbito educativo, ya que "la falta de un mecanismo efectivo que les permita escribir, leer o comunicarse se convierte en una seria barrera de acceso al conocimiento, a las posibilidades de autonomía y desarrollo personal" (Zappalá, 2018, p. 4); razón por la cual, son recursos que brindan soluciones ante barreras de aprendizaje y participación que se presentan durante el proceso formativo de cada individuo. 
Con respecto a lo anterior, García \& López (2012) mencionan que a lo largo del tiempo se ha presentado una evolución significativa en los procesos educativos, en el que el profesorado busca implementar las TIC como nuevos recursos que sirvan como apoyo para las PCD; pues éstas, han contribuido al proceso de inclusión al ofrecer alternativas tecnológicas que estimulen el desarrollo de las habilidades para la adquisición de nuevos conocimientos.

Sin embargo, para que se logre efectuar un proceso de mediación pedagógica exitoso es esencial que las TIC que se implementan en los centros educativos respondan a las características individuales de cada persona; aprovechando al máximo los recursos que estas herramientas le puedan brindar al estudiantado.

Bajo esta línea, es importante tomar en cuenta que existen muchas alternativas tecnológicas que favorecen el aprendizaje de cada individuo, por lo que los docentes deben buscar cuales son los apoyos o herramientas adecuadas según la condición de discapacidad de cada estudiante (Romero, Gonzáles, García \& Lozano, 2018). A continuación, se brindan algunos de ejemplos de TIC que pueden favorecer la superación de barreras en los centros educativos:

\subsubsection{Discapacidad intelectual}

Existen alternativas tecnológicas que pueden utilizar las PcD intelectual y que representan apoyos importantes en el desarrollo de sus habilidades y destrezas, como lo son las computadoras, tabletas, celulares, internet, software y hardware; herramientas que en la actualidad forman parte del diario vivir del estudiantado (García, 2014).

Estas herramientas tecnológicas pueden implementarse para incrementar la atención, motivación y destrezas relacionadas al área; así como recursos de apoyos para el acceso a un mundo de información y comunicación, que posibilitará la adquisición de nuevos conocimientos a esta población.

Asimismo, Sánchez (2013), menciona que la computadora "puede utilizarse de forma creativa para mejorar el desarrollo de habilidades y destrezas de los alumnos con discapacidad intelectual. Su carácter abierto y flexible favorece los diseños, proyectos y programaciones didácticas adaptadas a diferentes ritmos y tipos de aprendizajes" (p. 212); incrementando el acceso a diversas posibilidades de aprendizaje por parte del estudiantado.

Sin embargo, autores como Zappalá et al. (2011) establecen que si bien la computadora es una herramienta enriquecedora en los procesos de aprendizaje de este colectivo, las TIC que realmente colaboran en su formación son las aplicaciones, software y hardware que mantienen contenidos simples y accesibles; indicando a su vez, que es esencial "utilizar un lenguaje claro, no sobrecargar la pantalla con información, emplear íconos gráficos o lo suficientemente descriptivos para ayudar en la navegación, apoyos alternativos de comprensión a través de recursos auditivos, gráficos y/o de texto, etcétera" (p. 13), que favorezcan la comprensión de las temáticas tratadas.

Ante las barreras que presenta esta población, en cuanto a la presentación de contenidos en las herramientas tecnológica, se pueden emplear aplicaciones que contengan las especificaciones de diseño que mencionó Zappalá et al. (2011) anteriormente; algunas que se encuentran en Google Play son "Autonomía segura", "Entrenador de cerebro", "Smile and Learn: Juegos educativos para niños", "Visual Reading - educación especial", i“Talk UP!" "Communicator - Autismo" y "Entrena tu Cerebro Juegos de Atención".

Igualmente, programas como el Edilim y Tuxpaint "incluyen imágenes en movimiento, sonidos, textos estructurados y llamativos, en donde se expone al estudiante con discapacidad intelectual a diversas experiencias que lo alejan de la rutina, favoreciendo su motivación y por ende la adquisición de aprendizajes" (Sánchez, 2013, p. 216); lo que posibilita la superación de barreras de aprendizaje y el establecimiento de aprendizajes más significativos. 


\subsubsection{Discapacidad auditiva}

Las PcD auditiva habitualmente se enfrentan a barreras de comunicación, que impiden su desenvolvimiento exitoso en los diversos ambientes en los que se relaciona; en el ámbito educativo existen TIC que pueden ser implementadas como recursos y herramientas de apoyo para la superación de estos obstáculos.

Al respecto, Zappalá et al. (2011) consideran que trabajar utilizando fotografías e imágenes le permitirá al estudiantado "identificarse, describirse, reconocer a otros, narrar experiencias, secuenciar procesos" (p. 10); favoreciendo así la mediación pedagógica efectuada con personas sordas o que presentan baja audición, al generar un proceso formativo más adecuado y accesible, que asegure la participación del estudiantado en todas las actividades.

Asimismo, Sánchez (2013) indica que "la computadora e internet aparecen como una oportunidad que pueden aprovechar e integrar en su quehacer cotidiano. Les ayudará- por su versatilidad y potencia- al eliminar algunas dificultades de comunicación y les ofrecerá oportunidades de empleo, educación y bienestar social" (p. 220); por lo que el personal docente debe aprovechar estos recursos con el propósito de brindar los apoyos necesarios, para que esta población pueda enfrentar las barreras que se puedan presentar.

En la siguiente figura, se evidencian ejemplos de TIC propuestos por los autores Roig (2012), Luna (2013) y Rodríguez \& Arroyo (2014), que pueden favorecer el aprendizaje y desenvolvimiento del estudiantado con discapacidad auditiva en entornos formativos.

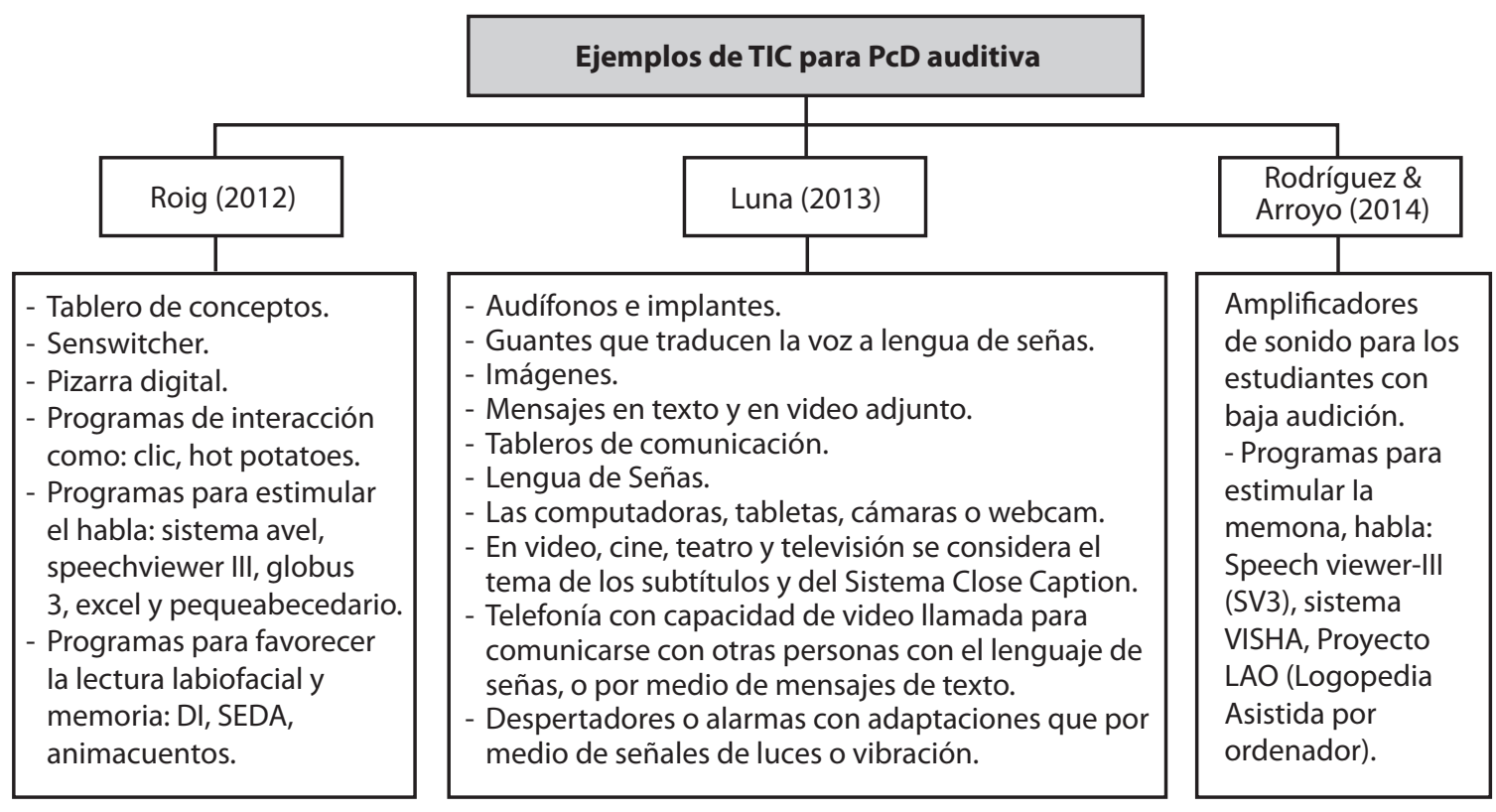

Figura 4. Ejemplos de TIC para el estudiantado con discapacidad auditiva.

Fuente: Elaboración propia basada en Roig (2012), Luna (2013) y Rodríguez \& Arroyo (2014).

Las herramientas tecnológicas que se visualizan en la figura anterior, fomentan la accesibilidad y motivación en las PcD, lo que ofrece mayores posibilidades de aprendizaje a este colectivo; igualmente, debido a que los programas son gratuitos, se convierten en recursos de fácil acceso para los centros educativos.

Por otra parte, existen aplicaciones a utilizar en tabletas o celulares, que actúan como apoyos para posibilitar la comunicación de estas personas, algunas de ellas son "Tablero de Comunicación", "Sordo 
ayuda", "Transcripción instantánea" y "Sordo-Mudo Ayudante", "Háblalo"; las cuales transmiten o transcriben conversaciones orales o gestuales.

De igual forma, aplicaciones como "Visualfy", sirven para que la persona sorda pueda reconocer en distintos ámbitos sonidos específicos, como alarmas de emergencia, campanas de salida o entrada a clases, avisos de lavadoras y microondas, implementando colores, destellos de luz o patrones de vibración para cada notificación (Visualfy, 2019).

Todas estas alternativas, son apoyos importantes para la mediación pedagógica de las PcD auditiva, ya que por medio de éstos se impulsa su autonomía; posibilitando así, el desarrollo de habilidades y destrezas que permiten su inclusión social y por ende el alcance de una mejor calidad de vida.

\subsubsection{Discapacidad visual}

Este colectivo debe enfrentar a diario barreras en el ámbito educativo, por lo que implementar TIC como apoyos puede brindar mayores oportunidades de aprendizaje; al respecto, Ribeiro \& Sánchez (2013) indican que el uso de computadoras con monitores de pantalla Braille, impresoras Braille, sintetizadores de voz, libros con formato electrónico y de sonido, software de Lector de Pantalla, de Reconocimiento Óptico de Caracteres (OCR) y de Magnificador de Pantalla, posibilitan el desenvolvimiento autónomo de estas personas.

Igualmente, Vázquez (2012) propone utilizar los procesadores de voz como programas o aplicaciones, pues "a través de un micrófono puede dictársele al ordenador cualquier tipo de documento (correos electrónicos o texto de todo tipo) [...] frecuentemente se utiliza Dragon Naturally Speaking y Via Voice" (p. 83). A partir de estas herramientas tecnológicas, el estudiantado con discapacidad visual puede utilizar una computadora, celular o tableta, facilitando la accesibilidad y posibilitando la superación de barreras; permitiéndole a esta población, participar activamente en su aprendizaje.

Asimismo, existen programas que convierten a las computadoras en herramientas más accesibles, como lo son los lectores de pantalla Voice Over o WebAnywhere para que las PcD visual puedan utilizar una computadora y el amplificador de pantalla Super Nova Reader Magnifier, el cual es una combinación de lector y magnificador de pantalla que propicia el acceso a las aplicaciones de Windows por medio de la ampliación de la imagen máxima de 32x aumentos, salida de voz o sistema braille (Herrando \& Coco, 2015).

Por otra parte, Herrando \& Coco (2015) también proponen un teclado llamado Braille Star, el cual "permite descargar documentos o texto desde un ordenador y almacenar la información, para más adelante disponer de ella e incluso modificarla y guardar esos cambios. Dispone de modelos con 40 u 80 caracteres" (p. 24); posibilitando a la persona con discapacidad visual, participar activamente en las actividades formativa. A continuación, en la figura 5 se muestra una serie de TIC como apoyos que se pueden utilizar con esta población para fortalecer su independencia. 


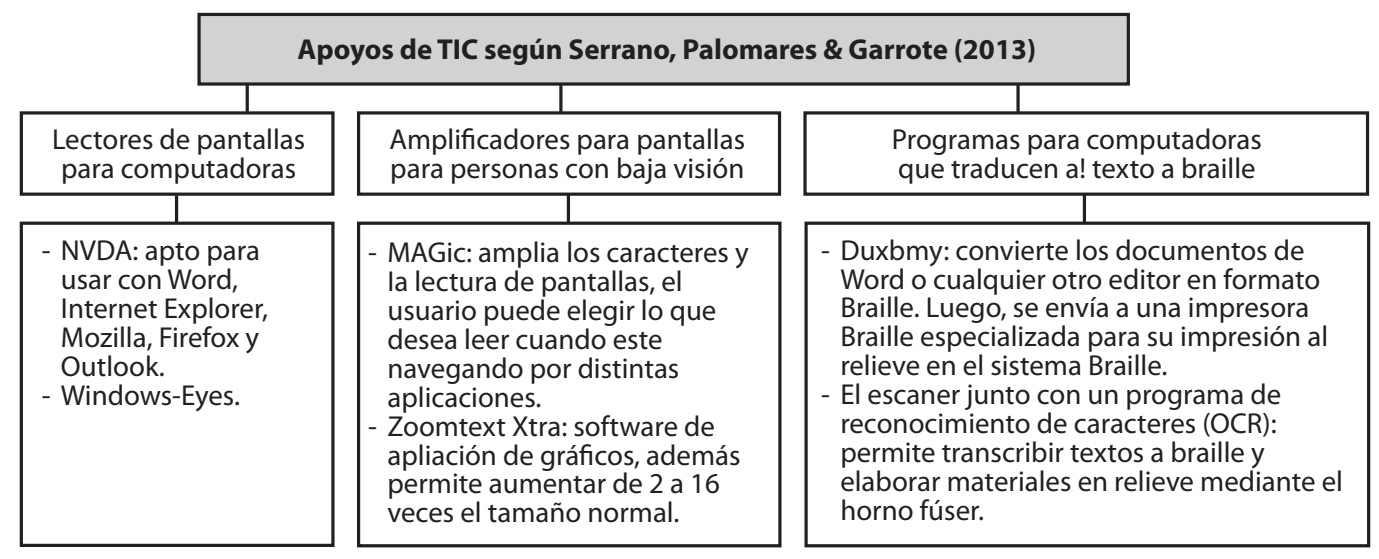

Figura 5. Apoyos de TIC para personas con discapacidad visual.

Fuente: Elaboración propia basada en Serrano, Palomares \& Garrote (2013).

Lo anterior, permite visualizar diferentes alternativas que pueden ser de mucha utilidad para las PcD visual, pues impulsan el desarrollo de su autonomía y colaboran en la construcción de su aprendizaje; propiciando el desarrollo de habilidades y competencias dirigidas al alcance de una mejor calidad de vida.

Según Herrando \& Coco (2015), existen múltiples aplicaciones para utilizarse en celulares y tabletas, las cuales serán presentadas en la siguiente figura.

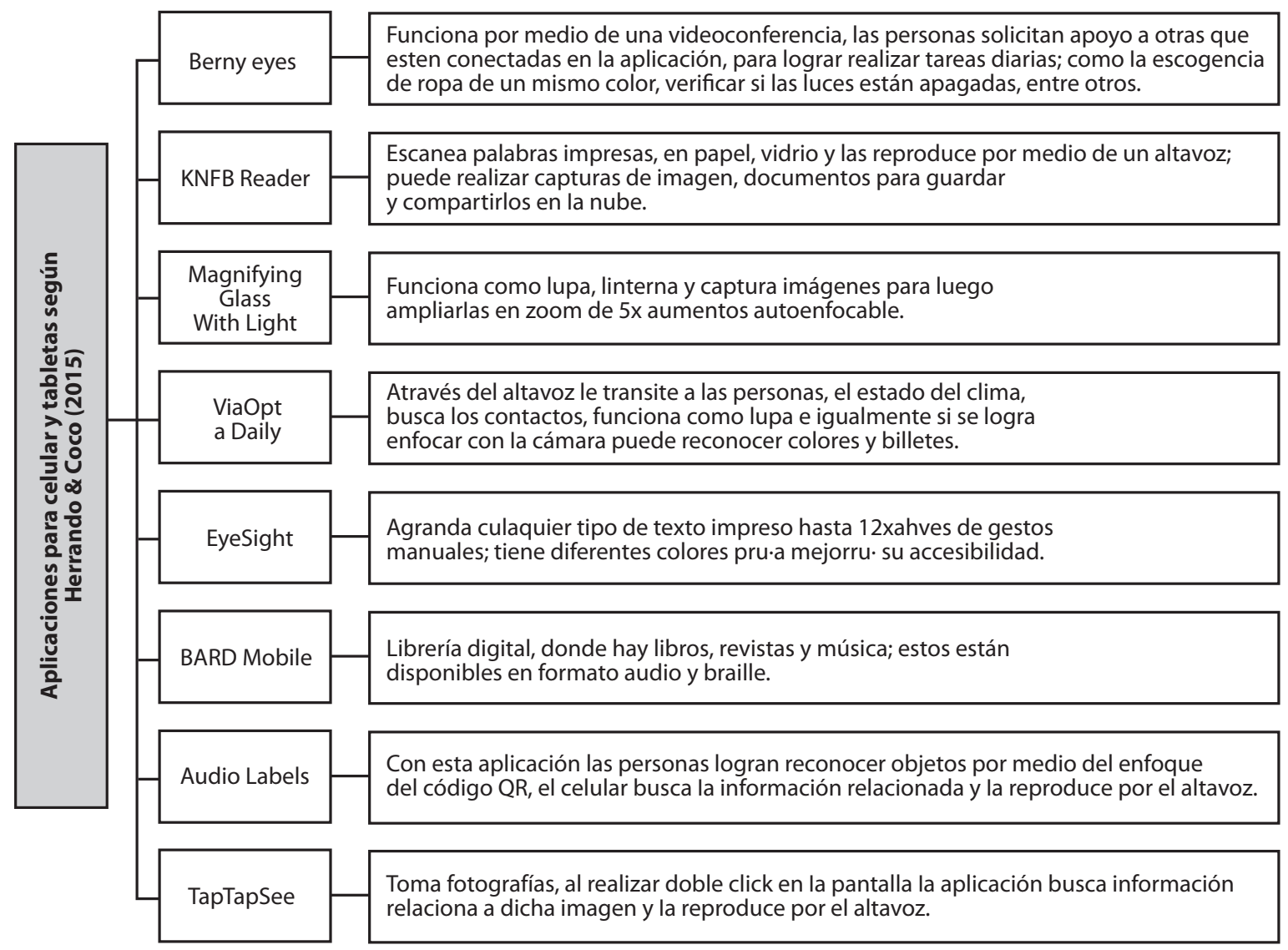

Figura 6. Aplicaciones para utilizarse en celulares y tabletas. Fuente: Elaboración propia basada en Herrando \& Coco (2015). 
Todas las aplicaciones mencionadas con anterioridad, son alternativas tecnológicas que podrán ser utilizadas en los centros educativos, con el objetivo de crear espacios accesibles para todo el estudiantado con discapacidad; eliminando así, barreras de aprendizaje y participación.

\section{Discapacidad física o motriz}

El estudiantado con discapacidad física día a día se enfrenta a barreras que obstaculizan su proceso de aprendizaje, como lo son "la accesibilidad en el entorno físico, la tecnología, las actitudes de la población frente a la discapacidad y los sistemas, servicios y políticas" (p. Serrano et al, 2013, p. 42); por lo que brindar apoyos dentro de las aulas propicia el autoaprendizaje y su desenvolvimiento autónomo.

Según Vázquez (2012), la computadora es una herramienta esencial para fomentar la participación de esta población, sin embargo establece que los sistemas de entrada de información al ordenador pueden representar barreras de acceso, pues algunas PcD motriz "no pueden acceder al teclado y ratón convencionales, que son los elementos más usuales de entrada y esto dificulta mucho el desarrollo de las competencias" (p. 80); por ello, es preciso utilizar TIC que faciliten la utilización de este recurso.

Bajo esta línea, las TIC son herramientas tecnológicas que posibilitan el acceso a las computadoras, esto al representar adaptaciones que garantizan la participación plena del estudiantado en los procesos de mediación pedagógica; los siguientes autores, demuestran algunas sugerencias de recursos que se pueden utilizar para lograr dicho propósito.

TABLA 2

TIC como apoyo para el acceso a computadoras

\begin{tabular}{|c|c|}
\hline Autores & Hardware para realizar las adaptaciones \\
\hline Rodríguez \& & - Soportes: atril que se puede apoyar en el reposa brazos de la silla de ruedas. \\
\hline Arroyo (2014) & $\begin{array}{l}\text { - } \quad \text { Conmutadores: pueden ser de soplo, de tacto, de inclinación y de palanca. } \\
\text { - } \quad \text { casilla viene dibujado un carácter. } \\
\text { - } \quad \text { Pantallas táctiles. } \\
\text { - } \quad \text { Adaptaciones en los ratones o mouses: como el TrackBall, teclado ergonómico, Joystick. }\end{array}$ \\
\hline Muñoz (2014) & $\begin{array}{l}\text { - Teclados alternativos: modifica la velocidad de repetición de las teclas, interruptores, punteros, carcasas, } \\
\text { programas de reconocimiento de voz, controles remotos para el desplazamiento de silla. }\end{array}$ \\
\hline $\begin{array}{l}\text { Romero et al. } \\
(2018)\end{array}$ & $\begin{array}{l}\text { - Teclados reducidos, ampliados, para una sola mano, por pedales, de carcasa, de conceptos, licornio. } \\
\text { - Mouse o ratones: ratón controlado por teclas, ratón externo de barrido, joystick, emulador de ratón por } \\
\text { pulsadores, ratón para barbilla y ratón por sensores ópticos. }\end{array}$ \\
\hline
\end{tabular}

Fuente: Elaboración propia basada en Rodríguez \& Arroyo (2014), Muñoz (2014) y Romero et al. (2018).

Lo anterior, evidencia herramientas enriquecedoras que permiten desarrollar habilidades y destrezas tecnológicas; posibilitando a las PCD física la utilización la computadora con libertad y brindando los apoyos necesarios para que los individuos puedan ser independientes en el momento de enfrentar las diferentes barreras que se le presenta a este colectivo.

Es importante mencionar, que existen aplicaciones didácticas específicas para celulares, tabletas o computadoras que facilitan el acceso y la comunicación de este colectivo, entre ellas se encuentran "Kanghooru", "Screen Scanner", "Boardmaker", "Plaphoons", "ARASAAC", "Supernova", "Open Book", "Preditext", "Lamosqueta.cat", "Plates volar" y "Actividades zonaclic"; todas estas herramientas interactivas, favorecen la adquisición de aprendizaje del estudiantado (Romero et al., 2018). 
Ante la gran variedad de TIC mencionadas con anterioridad, es fundamental que se valore cuál es la que mejor se adecue a las características de cada persona; pues éstas, pueden ser utilizadas en los centros educativos con el objetivo de crear espacios accesibles para todo el estudiantado, donde se brinden los apoyos necesarios para que cada uno pueda ser partícipe de su propio aprendizaje y enfrentar las barreras que se puedan presentar.

\section{CONCLUSIONES}

Esta revisión bibliográfica evidencia los aportes que trae la aplicación de las TIC al implementarlas como recurso de apoyo para las PCD; evidenciando, la forma en que estas tecnologías brindan las herramientas necesarias a cada individuo para enfrentar las barreras de aprendizaje y participación que se puedan presentar en su proceso de desarrollo.

A su vez, a partir de investigaciones como las de Zappalá et al. (2011), García \& López (2012), Ribeiro \& Sánchez (2013), Rodríguez \& Arroyo (2014), Muñoz (2014) y Rodríguez (2015), se logra comprobar la importancia de las TIC como herramientas de apoyo para que las PCD puedan desarrollar integralmente las competencias dirigidas a la superación de barreras y al desenvolvimiento autónomo de esta población.

Asimismo, estos autores posibilitan la identificación de las TIC como medios para el ofrecimiento de oportunidades de acceso e incorporación al contexto social, educativo y laboral del colectivo con discapacidad; siendo así instrumentos para el ejercicio de los derechos y deberes que como seres humanos les pertenece.

Un objetivo primordial de la educación dirigida a las PcD, es el estímulo de competencias dirigidas a su empoderamiento para la toma de decisiones y al desenvolvimiento autónomo en diversos entornos; por lo que el utilizar las TIC en la educación es sin lugar a duda una forma significativa de inclusión, en la que se promueve la accesibilidad y la construcción de una sociedad más activa.

Bajo esta línea, la información presentada por autores como Valencia et al. (2016), García (2014), Rodríguez (2015), Sánchez (2013) y Marqués (2012), permiten afirmar que las nuevas tecnologías son un medio necesario para implementar en los centros educativos; incluyéndolas en las diferentes metodologías de aprendizaje para ampliar las oportunidades de adquisición de conocimientos y estimular el interés, motivación, pensamiento crítico y el aprendizaje por descubrimiento de las PcD.

Igualmente, Zappalá (2018), García y López (2012) y Romero et al. (2018) evidencian la importancia de efectuar una valoración para la selección de TIC de acuerdo con la condición de discapacidad que presenta cada persona, pues de ello dependerá el éxito en la adquisición de aprendizajes.

Ante esto, es fundamental que el profesorado aproveche la gran variedad de TIC existentes en el mercado, para generar procesos de mediación pedagógica orientados a responder a las características individuales de cada estudiante; posibilitando su inclusión en las aulas y por ende construyendo entornos asertivos y equitativos que respeten la diversidad.

Para ello, es elemental que se tomen en cuenta las sugerencias de TIC brindadas por los autores García (2014), Sánchez (2013), Zappalá et al. (2011), Roig (2012), Luna (2013), Rodríguez \& Arroyo (2014), Ribeiro \& Sánchez (2013), Vásquez (2012), Herrando \& Coco (2015), Serrano et al. (2013), Muñoz (2014) y Romero et al. (2018); ya que estas recomendaciones le brindan importancia a la implementación de herramientas tecnológicas como computadoras, telefonía móvil, teclados, mouse, aplicaciones, software, hardware, entre otros, para el acceso a factores contextuales y conceptuales.

En conclusión, implementar diversas TIC dentro del entorno educativo ha demostrado un avance en el proceso de aprendizaje de esta población; pues, su gran variedad tecnológica permite adaptar diversas 
herramientas a las características de cada persona, dando paso al desarrollo de habilidades y destrezas de acuerdo con los intereses de cada individuo y al fortalecimiento de competencias necesarias para la superación de barreras de aprendizaje y participación.

\section{REFERENCIAS}

Campaña Mundial por la Educación. (2013). Igualdad de derechos, igualdad de oportunidades. La educación inclusiva para niños con discapacidad. Recuperado de http://www.campaignforeducation.org/ docs/reports/Equal\%20Right\%20Equal\%200pportunity\%20ES.pdf.

De la Torre, L. \& Domínguez, J. (2012). Las TIC en el proceso de enseñanza aprendizaje a través de los objetos de aprendizaje. Revista Cubana de Informática Médica, 4(1), 91-100. Recuperado de http:// scielo.sld.cu/pdf/rcim/v4n1/rcim08112.pdf

Fernández, J., Reyes, M., \& El Homran, M. (2018). TIC y discapacidad. Principales barreras para la formación del profesorado. Revista de Educación Mediática y TIC, 7(1), 1-25. Recuperado de https://dialnet. unirioja.es/descarga/articulo/6382219.pdf

Fundación Juan Ciudad. (2016). Voluntariado con Cuadernos formativos Personas con Discapacidad Intelectual. Recuperado de https://www.uma.es/media/tinyimages/file/Voluntariado_con_ Personas_con_Discapacidad_Int.pdf

García, M. (2014). Las TICs aplicadas a las Necesidades Educativas Especiales. (Trabajo Final de Grado en Educación Primaria). Recuperado de https://uvadoc.uva.es/bitstream/10324/8376/7/TFG-O\%20 376.pdf

García, M. \& López, R. (2012). Explorando, desde una perspectiva inclusiva, el uso de las TIC para atender a la diversidad. Revista de Currículum y Formación de Profesorado, 16(1), 277-293. Recuperado de https://www.redalyc.org/pdf/567/56724377016.pdf

Herrando, J. \& Coco, R. (2015). Papel de las TIC en personas con baja visión. (Tesis de maestría en rehabilitación visual Universidad de Valladolid). Recuperado de https://uvadoc.uva.es/bitstream/10324/14175/1/TFM-M243.pdf

Luna, M. (2013). Tecnología y discapacidad: Una mirada pedagógica. Revista Digital Universitaria, 12(14), 1-19. Recuperado de http://www.revista.unam.mx/vol.14/num12/art53/art53.pdf

Marqués, P. (2012). Impacto de las TIC en la educación: funciones y limitaciones. Recuperado de https:// www.3ciencias.com/wp-content/uploads/2013/01/impacto-de-las-tic.pdf

Molina, K. \& Cuevas, F. (2014). TIC y Educación de Personas con Discapacidad en Costa Rica. Recuperado de https://www.google.com/url?q=https://www.oei.es/historico/congreso2014/memoriactei/1202. pdf\&sa=D\&ust=1558039029919000\&usg=AFQjCNHuZRpN5gSj83J_W-ZLWeWhZ7j_Ew

Muñoz, P. (2014). Inventario de recursos TIC para niños con necesidades educativas especiales. (Tesis de grado para optar por el título de Psicóloga Educativa en el grado académico de Licenciada). Recuperado de http://biblio3.url.edu.gt/Tesario/2014/05/24/Munoz-Paula.pdf

Poder Ejecutivo de Costa Rica. (2018). Decreto Ejecutivo N 41088-MP. Recuperado de http://www. pgrweb.go.cr/scij/Busqueda/Normativa/Normas/nrm_texto_completo.aspx?param1=NRTC\&nValor1=1\&nValor2=86570\&nValor3=112389\&param2=1\&strTipM=TC\&IResultado=1\&strSim=simp

Ribeiro, J. \& Sánchez, S. (2013). Inclusión Educativa a través de las TIC. Indagatio Didactica, 5(4), 147-160. Recuperado de https://www.academia.edu/31858339/ Inclusi\%C3\%B3n_Educativa_a_trav\%C3\%A9s_de_las_TIC

Rodríguez, C. (2015). Uso de las TIC para favorecer el proceso de aprendizaje de estudiantes con Discapacidad Intelectual en la Institución Educativa Nicolás Gómez Dávila, Bogotá, Colombia. Estudio de caso. 
(Tesis de maestría). Recuperado de https://repositorio.itesm.mx/bitstream/handle/11285/626577/ Claudia_Rodr\%C3\%ADguez_Barrera_.pdf?sequence=1

Rodríguez, M. \& Arroyo, M. (2014). Las TIC al servicio de la inclusión educativa. Digital Education Review, (25), 108- 126. Recuperado de https://dialnet.unirioja.es/servlet/articulo?codigo=4778259

Romero, S., Gonzáles, I., García, A. \& Lozano, A. (2018). Herramientas tecnológicas para la educación inclusiva. Revista tecnológica- ciencia y educación, (9), 83- 112. Recuperado de http://www.tecnologia-ciencia-educacion.com/index.php/TCE/article/download/175/148

Roig, M. (2012). Utilización de recursos TIC para la integración del alumnado con discapacidad auditiva en primaria. (Trabajo final de grado en maestro de educación primaria). Recuperado de https:// reunir.unir.net/bitstream/handle/123456789/862/2012_09_17_TFG_ESTUDIO_DEL_TRABAJO. pdf?sequence $=1 \&$ isAllowed $=y$

Sánchez, R. (2013). Itinerarios Inclusivos, Computadora y Competencias. Costa Rica: EUNED.

Serrano, C., Ramírez, C., Abril, J., Ramón, L., Guerra, L. \& Clavijo, N. (2013). Barreras contextuales para la participación de las personas con discapacidad física. Salud UIS, 45 (1), 41-51. Recuperado de http:// www.scielo.org.co/pdf/suis/v45n1/v45n1a06.pdf

Serrano, I., Palomares, A. \& Garrote, D. (2013). Propuestas innovadoras para favorecer el uso de las TIC y propiciar la inclusión educativa del alumnado con discapacidad visual. Revista de la Facultad de Educación de Albacete, (28), 61-74. Recuperado de https://dialnet.unirioja.es/descarga/articulo/4911349.pdf

Valencia, T., Serna, A., Ochoa, S., Caicedo, A., Montes, J. \& Chávez, J. (2016). Competencias y estándares TIC desde la dimensión pedagógica. Recuperado de http://www.unesco.org/new/fileadmin/ MULTIMEDIA/FIELD/Santiago/pdf/Competencias-estandares-TIC.pdf

Vásquez, A. (2015). La Educación Inclusiva: El cambio sostenible que requiere la educación costarricense. Revista CENAREC para Todos, (6), 1-54. Recuperado de http://www.cenarec.go.cr/index.php/ component/jdownloads/send/14-revistas/51-iiedicion2015? Itemid=0

Vásquez, E. (julio, 2012). Propuesta de un inventario de recursos tecnológicos para el tratamiento del alumnado con discapacidad en el Espacio Europeo de Educación Superior. Dialnet, 20, 71-92. Recuperado de https://dialnet.unirioja.es/descarga/articulo/4105066.pdf

Victoria, J. (2013). El modelo social de la discapacidad: una cuestión de derechos humanos. Revista de derecho UNED, 12, 817-833. Recuperado de http://revistas.uned.es/index.php/RDUNED/article/ viewFile/11716/11163

Visualfy. (2019). Visualfy - Ayuda a Personas Sordas. Recuperado de https://play.google.com/store/apps/ details?id=com.fusiodarts.visualfy.lite

Zappalá, D. (2018). Inclusiva. "Construyendo sociedades inclusivas. Recuperado de https://www.dropbox.com/s/ih7ld3p2jmgzkwx/12\%20-\%20Revista\%20Inclusiva-CENAREC-Tecnolog\%C3\%ADaDiciembre-2018.pdf?dl=0

Zappalá, D., Köppel, A. \& Suchodolski, M. (2011). Inclusión de tic en escuelas para alumnos con discapacidad intelectual. Recuperado de http://escritorioeducacionespecial.educ.ar/datos/recursos/pdf/m-intelectuales-1-40.pdf

Zappalá, D., Köppel, A. \& Suchodolski, M. (2011). Serie de estrategias pedagógicas: Inclusión de TIC en escuelas para alumnos con discapacidad intelectual. Recuperado de https://es.calameo.com/ $\mathrm{read} / 00426078351 \mathrm{~cd} 0 \mathrm{~b} 418 \mathrm{ab} 7$ 